Portfolio

\title{
Sciences, Arts et Progrès! Une visite au musée Spitzner en 1895
}

Science, Art and Progress! A Visit to the Spitzner Museum in 1895

\section{Anne Carol et Béatrice Hermitte}

\section{(2) OpenEdition}

\section{Journals}

Édition électronique

URL : https://journals.openedition.org/aes/4305

DOI : $10.4000 /$ aes. 4305

ISSN : 2258-093X

Éditeur

Laboratoire LISAA

Référence électronique

Anne Carol et Béatrice Hermitte, "Sciences, Arts et Progrès! Une visite au musée Spitzner en 1895 », Arts et Savoirs [En ligne], 16 | 2021, mis en ligne le 17 décembre 2021, consulté le 19 décembre 2021. URL : http://journals.openedition.org/aes/4305 ; DOI : https://doi.org/10.4000/aes.4305

Ce document a été généré automatiquement le 19 décembre 2021.

Centre de recherche LISAA (Littératures SAvoirs et Arts) 


\title{
Portfolio
}

\section{Sciences, Arts et Progrès! Une visite au musée Spitzner en 1895}

Science, Art and Progress! A Visit to the Spitzner Museum in 1895

\author{
Anne Carol et Béatrice Hermitte
}

\section{NOTE DE L'ÉDITEUR}

Cet article contient des images susceptibles de heurter la sensibilité de certains lecteurs

\section{Introduction}

1 Le musée Spitzner est l'un des musées anatomiques et forains les plus renommés de son époque. Fondé et dirigé par Pierre Spitzner (Stahlberg, 1833 ; Gand, 1896), il ouvre ses portes en 1856 à Paris, dans le pavillon de la Ruche place du Château d'Eau, actuelle place de la République. Après 1870 , au moment où les musées anatomiques connaissent leur apogée sur les champs de foire, il devient en partie itinérant, s'exposant en Europe et aux États-Unis ${ }^{1}$, avant de se fixer à Bruxelles en 1934. Peu à peu déserté après la deuxième guerre mondiale, il ferme ses portes au début des années 1960. Redécouvertes en 1979 par la journaliste Margo Bruynoghe, les collections échappent de peu à la dispersion lors d'une vente à Drouot en 1985. Une partie d'entre elles est actuellement exposée au musée de médecine de Montpellier².

2 Le musée aurait présenté à ses visiteurs jusqu'à 6500 pièces selon les affiches et tracts édités à des fins publicitaires. Ses collections étaient principalement constituées de pièces en cire $^{3}$, d'un réalisme troublant, et d'artefacts anatomiques (séchés ou conservés dans de l'esprit de vin). Le droit d'entrée assez modique, de 50 centimes, devait permettre au plus grand nombre de le visiter. D'abord ouvert aux hommes, son accès est autorisé aux femmes dans les années 1880, sauf aux femmes enceintes, en 
raison des malaises que la vue de certaines pièces pouvait susciter. Entre 1856 et la fermeture du musée dans les années 1960, les collections évoluent au gré du contexte sanitaire, des progrès scientifiques, de l'histoire, voire des faits divers. Selon les époques, leur périmètre englobe ainsi l'anatomie normale, l'anatomie pathologique, l'ethno-anthropologie, l'histoire de la médecine et de la chirurgie, la botanique... Seules les pièces d'anatomie normale et pathologique, noyau du musée, furent présentées continûment durant les cent-vingt années d'existence du musée.

3 Pierre Spitzner fait partie de ces «entrepreneurs de spectacles scientifiques » qui émergent à partir du XVIII ${ }^{\mathrm{e}}$ siècle et s'épanouissent à l'âge industriel. Daniel Raichvarg relève une série de critères communs à ces spectacles : « l'ouverture sur un public très large, le poids de l'actualité dans les réalisations, une vocation pédagogique toujours présente conjuguée à une non moins présente envie de merveilleux ${ }^{4}$ ». L'exposition de ces collections se fondait de fait sur un impératif didactique, gage de sa respectabilité. Le catalogue édité en 1895, le plus ancien conservé, portait en exergue deux citations : «Homme, apprends à te connaître (Ambroise Paré) » et "l'Étude fait le progrès (Darwin)». Au même moment, un journaliste reconnaissait que les collections présentées par le musée étaient " horribles », mais que le musée méritait « d'être vu et même revu $»^{5}$. Le musée devait initier le public à la complexité de la machine humaine et de son fonctionnement; il prolongeait ainsi la conception de l'anatomie comme une science destinée à mettre en lumière les merveilles de la création ${ }^{6}$. L'exploration du monde et la colonisation lui fournirent l'occasion d'étendre ses visées pédagogiques à l'anatomie comparée et à l'anthropologie raciale, le transformant en «Grand musée anatomique anthropologique et ethnologique ». Cette fonction instructive se doublait d'une fonction édifiante. Les maladies et les lésions présentées devaient en effet sensibiliser les visiteurs aux notions d'hygiène, et contribuer à lutter particulièrement contre quatre fléaux sociaux qui inquiétaient médecins et démographes à la fin du XIX siècle : la syphilis, la tuberculose, l'alcoolisme, et la mortalité infantile. Par ailleurs, dans un contexte où les «merveilles de la science » ou « les merveilles de l'industrie » faisaient l'objet d'encyclopédies illustrées ${ }^{7}$ et où les expositions universelles offraient à l'Occident un miroir où s'admirer, le musée mettait aussi en scène les progrès et les triomphes de la médecine. Mais s'en tenir à ces nobles objectifs ne suffirait pas à expliquer son succès. Le musée Spitzner avait aussi une dimension récréative, qu'il partageait avec d'autres attractions foraines contemporaines, avec lesquelles il voisinait sur les champs de foire : entresorts, freak shows ou montagnes russes ${ }^{8}$; comme eux, il procurait - intentionnellement ou pas ? - des sensations fortes, d'ordre visuel du moins. En tant que tel, il a durablement marqué des générations de visiteurs, dont il a contribué à façonner le regard, y compris dans sa dimension esthétique. Il est banal d'évoquer ici son influence sur le jeune Paul Delvaux qui représente de façon récurrente les squelettes et les Vénus qui s'exposaient à l'entrée9 .

4 Aujourd'hui, le regard porté sur les collections Spitzner court le risque de deux oblitérations. Il faut en premier lieu se débarrasser d'une lecture décliniste et condescendante de la céroplastie sur les champs de foire, justement pointée par Chloé $\operatorname{Pirson}^{10}$. Selon ce scénario, écrit à la fin du XIX ${ }^{e}$ siècle, la céroplastie artistique et scientifique des $\mathrm{XVIII}^{\mathrm{e}}$ et du début du XIX $\mathrm{X}^{\mathrm{e}}$ siècle aurait trahi ses nobles missions et aurait prostitué la science auprès d'un public surtout avide de nudités pornographiques et de gore. Comme on le verra, le musée Spitzner a gardé une vocation pédagogique jusqu'à la deuxième guerre mondiale, et le sentiment d'étrangeté qu'il procure 
aujourd'hui ne doit pas occulter son projet savant. L'interprétation des collections pose un autre problème ; à l'instar des photographies scientifiques parfois présentées sans médiation au milieu d'œuvres d'art ${ }^{11}$, elles ne devraient pas être séparées du contexte de leur fabrication et des fonctions documentaires qui leur ont été assignées. La plupart des cires Spitzner sont produites pendant le deuxième âge de la céroplastie, au début $\mathrm{du} \mathrm{XIX}^{\mathrm{e}}$ siècle, lorsque celle-ci entend rompre, justement, avec les ambitions esthétisantes de la production antérieure et aspire à une objectivité maximale ${ }^{12}$. Si la devise du musée «Arts, sciences, progrès!» renvoie à l'art de la céroplastie, notre regard contemporain a tendance à inclure aussi dans ces « arts » le répertoire de motifs artistiques qui traversent, en amont et en aval, le spectacle du musée. Or, si le visiteur ordinaire n'était pas nécessairement en mesure de replacer les collections dans une généalogie visuelle, a fortiori était-il incapable de les relier à des productions postérieures ${ }^{13}$. En d'autres termes, l'anachronisme et la surinterprétation menacent, lorsqu'on essaie de comprendre quelles étaient les perceptions des spectateurs du XIX siècle.

Celles-ci nous sont malheureusement inaccessibles, car les témoignages sont rares et situés au $\mathrm{xx}^{\mathrm{e}}$ siècle $^{14}$, alors même que le regard est décalé par rapport aux objectifs originels que le musée poursuivait. Dans ces circonstances, l'historien ne peut que tenter de reconstituer les logiques à l'œuvre dans la présentation de telle ou telle pièce, la contextualiser dans les collections et au-delà, dans d'autres spectacles contemporains, et s'interroger sur ses échos potentiels dans les savoirs, les sensibilités et les cultures esthétiques des visiteurs d'hier à aujourd'hui.

Pour toutes ces raisons, les images qui composent ce portfolio ont été choisies dans le souci d'approcher, à l'instar du visiteur, les collections du musée dans leur globalité, leur cohérence et leur organisation, et seront mises en relation avec le catalogue qui les accompagnait. Compte tenu des variations des catalogues successifs (qui suivent celles du périmètre des collections), nous avons choisi de nous appuyer sur le catalogue de 1895 pour proposer cette visite guidée du musée $e^{15}$. 
Fig. 1

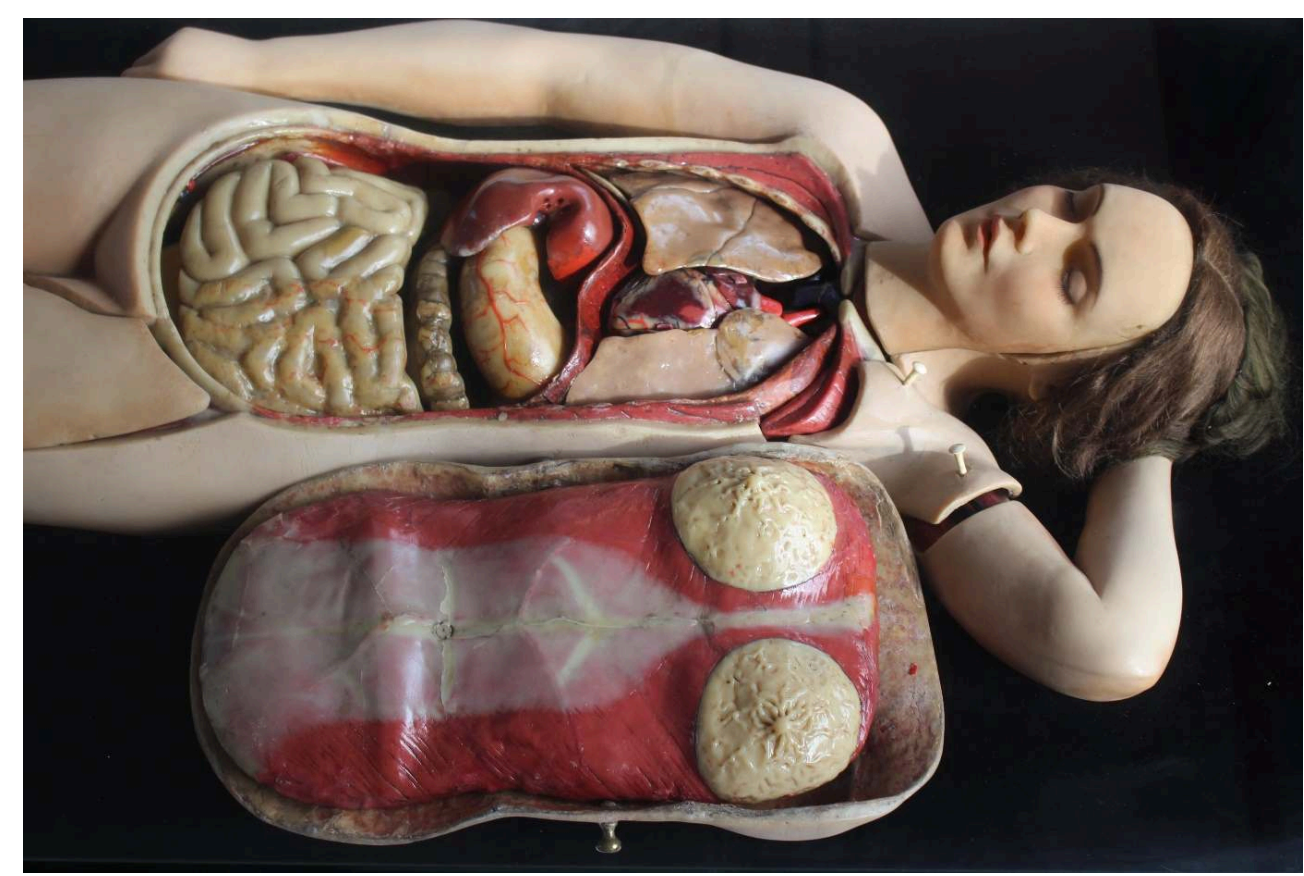

Vénus anatomique. xix ${ }^{\mathrm{e}}$ siècle. Cire colorée, cheveux naturels, yeux en verre soufflé.

Collection Pierre Spitzner. Classement en 2004 au titre des monuments historiques, Faculté de médecine de l'Université de Montpellier (Inv. UM.ANAT.DOR. 1214)

(c) Université de Montpellier

7 Une des pièces maitresses des musées d'anatomie, qu'ils soient académiques ou forains, était la Vénus anatomique. La Vénus anatomique était un mannequin en cire grandeur nature, dotée de cheveux et de cils naturels, dont on pouvait démonter les parties superficielles du tronc, de la face et de la cuisse pour faire apparaître les tissus et les viscères sous-jacents, en les retirant successivement. Il existait deux versions masculines moins sophistiquées de la Vénus anatomique dans le musée: un «homme complet» dont la peau et une portion du tronc pouvaient s'enlever "pour voir la construction intérieure du corps humain » de la gorge au rectum, ainsi qu'un «corps d'homme ouvert par le dos » qui était «en montre à l'extérieur », mais n'était pas démontable.

8 Les premières Vénus anatomiques en cire ont été fabriquées au XVIII siècle, dans le cadre de collections médicales ${ }^{16}$. La plus célèbre est celle réalisée par Susini et exposée à la Specola, le musée d'anatomie de Florence ouvert en 1775. Sa fonction était double : il s'agissait de suppléer aux dissections, en offrant un matériau pédagogique stable et dénué de tout aspect répugnant; mais aussi de donner à voir au public, admis gratuitement, les merveilles de la plus achevée des créations divines. Ces deux impératifs imposaient de fabriquer un mannequin dont la beauté et la sérénité neutralisaient la cruauté de l'éviscération. La Vénus italienne reposait donc sur un lit luxueux, les bras mollement allongés le long du corps, le cou entouré d'un collier de perles, le visage légèrement renversé en arrière, yeux et bouche entrouverts, donnant ainsi l'impression de participer en pleine conscience et sans souffrance à la révélation des mystères de la vie.

9 La Vénus de Spitzner relève du même objectif pédagogique, mais de façon plus sobre et, pour ainsi dire, désenchantée. Si elle repose dans une pose plus élaborée que la Venus 
florentine, un bras replié derrière la tête et les chevilles entrecroisées, ses yeux sont clos (ce qui lui vaut parfois le nom de Venus endormie), comme si elle était absente de la scène dont elle est le personnage principal, comme si elle en était l'objet et non plus le sujet. Ou peut-être est-elle morte, ce qui ramènerait la scène à la simple restitution d'une dissection réelle, juste euphémisée.

Le directeur et guide du musée procédait au démontage commenté de la Vénus à des horaires fixes. Dans la mesure où depuis la fin de l'époque moderne la dissection, n'était plus publique, mais enfermée dans les amphithéâtres, le musée Spitzner offrait donc à ses visiteurs une opportunité de s'initier à un ersatz de travaux anatomiques. La décomposition de cette figure devait expliquer aux visiteurs l'anatomie et le fonctionnement normal du corps humain, dans une sorte de performance démonstrative qui s'inscrit dans une longue tradition alliant science et théâtralité ${ }^{17}$. Cette fragmentation devait aussi préparer le spectateur à ce qu'il allait voir, c'est-à-dire des fragments de corps et des organes alignés dans des vitrines, en bonne santé ou malades. Ce morcellement marquait l'aboutissement d'un processus de transformation du regard sur le corps depuis la remise en cause au xvII ${ }^{\mathrm{e}}$ siècle de la médecine humorale, axée sur les fluides circulant dans le corps entier, et d'une attention croissante à ses parties solides, fibres, tissus, organes déclinés dans des Atlas anatomiques selon des échelles de plus en plus fines.

Fig. 2

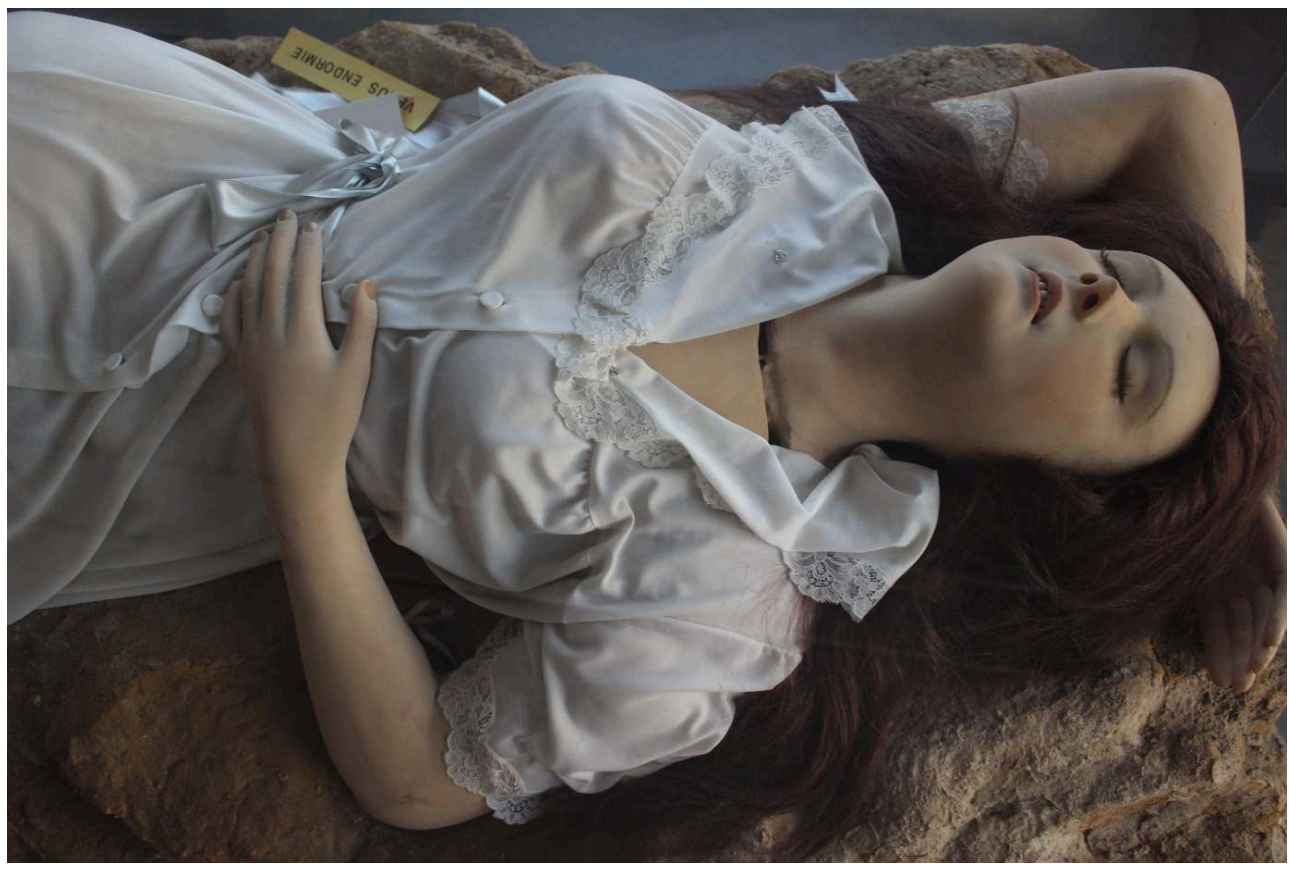

Vénus au repos. $x \mid x^{\mathrm{e}}$ siècle. Cire colorée, textile, cheveux naturels, yeux en verre soufflé. Collection Pierre Spitzner. Classement en 2004 au titre des monuments historiques. Faculté de médecine de I'Université de Montpellier (Inv. UM.ANAT.DOR. 1476)

(c) Université de Montpellier

La Vénus anatomique était doublée au musée Spitzner d'une autre attraction : la Vénus au repos, qui attirait le chaland sur le perron. Il s'agit aussi d'un mannequin de femme en cire, mais revêtue d'une chemise blanche, curieusement posée sur un pseudo socle rocheux. Dans sa poitrine un mécanisme électrique avait été inséré afin d'imiter le 
mouvement de la respiration, en soulevant sa chemise à intervalles réguliers. Le premier modèle animé de ce type avait été présenté par Curtius dans son cabinet de cires parisien en 1767, à côté de bustes de célébrités. La «belle endormie " du musée Spitzner est la réplique de celle du Docteur Lauret - qui avait lui-même ouvert un Grand musée français d'anatomie et pathologie à Paris en 1865, et qui avait «obtenu deux médailles à l'Exposition de Vienne (Autriche) [...] Ce chef-d'œuvre surpasse tout ce que l'on a pu obtenir jusqu'à ce jour et renferme à lui seul ces trois mots : ART, SCIENCE, PROGRÈS. $»^{18}$ En réalité, la présence de la Vénus endormie dans les collections ne se justifiait pas tant par sa valeur médico-pédagogique, quasi nulle, que par la fascination suscitée par la puissance mimétique de la cire et du mécanisme, et l'atmosphère vaguement érotique de ce corps abandonné aux yeux des spectateurs, « modelé d'après nature $»$.

Vénus anatomiques et Vénus au repos partageaient une généalogie complexe. Outre leur registre scientifique et leur fonction primitive de dévoilement des mystères de la nature, elles s'inscrivaient dans une culture visuelle religieuse. Les «cercueils de verre » qui les protégeaient rappelaient certaines châsses des saintes exposées dans les églises; celles-ci contenaient des artefacts céroplastiques intégrant des reliques, qui suggéraient l'imputrescibilité du corps saint. La pose tourmentée des Vénus, leur tête renversée ou leurs yeux entrouverts rappelaient aussi parfois les représentations des extases comme L'extase de Sainte-Thérèse du Bernin (1647-1652), que Charcot qualifie d'hystérique au même moment. Ce n'est pas le cas des modèles de Spitzner, qui semblent simplement plongées dans le sommeil, et livrent ainsi au public sa seminudité pour l'une, son ultra-nudité pour l'autre ${ }^{19}$. Leur posture renvoie plutôt au genre profane et mythologique des Vénus endormies dont le corps nu s'offre aux regards, de Giorgone à Titien. Or ce genre continue à s'exposer au même moment, par exemple au Salon de 1863, qualifié par Théophile Gautier de "Salon des Vénus ", car Alexandre Cabanel, Paul Baudry et Eugène-Emmanuel Amaury-Duval y exposaient leur interprétation du mythe de la naissance de Vénus. Celle de Paul Baudry, présentée au Salon sous le titre de La Perle et la Vague ${ }^{20}$ n'est pas sans évoquer la Vénus au repos de la collection Spitzner. Le mannequin est aussi allongé sur un rocher, dans une position quasi-identique. Celle de Cabanel, dont la posture contorsionnée est plus proche encore, fut particulièrement critiquée, car certains y voyaient une évocation du monde de la courtisanerie ${ }^{21}$ - en attendant l'olympia de Manet.

Vidée de sa fonction d'enchantement, la Vénus est donc investie d'une charge érotique. Mais c'est l'œil du visiteur qui en change la perception, malgré les précautions prises par leur modeleur pour pacifier l'expression de ses modèles et les désexualiser (la Vénus anatomique de Spitzner n'a ni pilosité, ni fente génitale). 


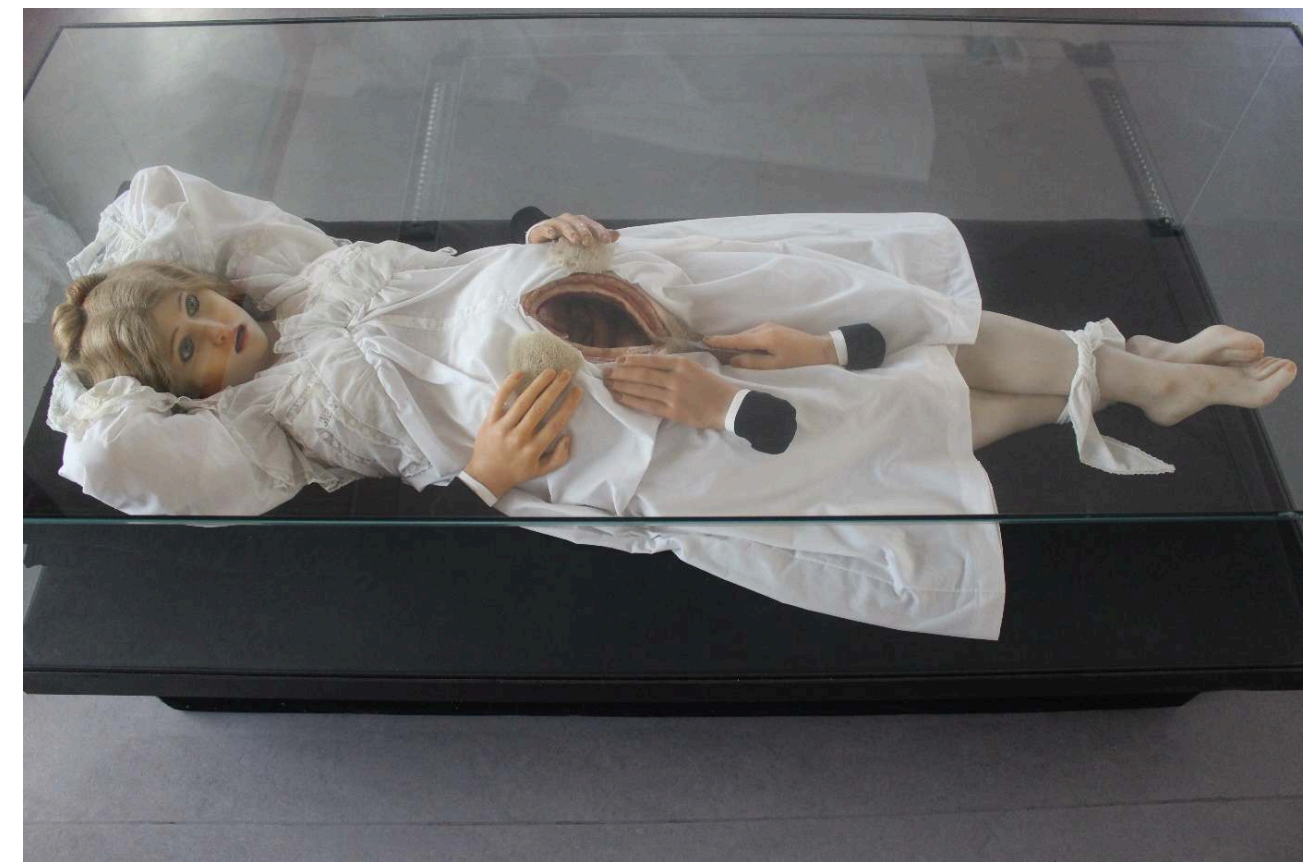

Opération de la césarienne. xıx siècle. Cire colorée, textile, cheveux naturels, yeux en verre soufflé. Collection Pierre Spitzner. Classement en 2004 au titre des monuments historiques. Faculté de médecine de l'Université de Montpellier (Inv. UM.ANAT.DOR. 1281).

(c) Université de Montpellier

Le musée Spitzner présentait une section entière consacrée aux accouchements et à la chirurgie obstétricale ; celle-ci s'intégrait à un ensemble plus vaste, voué à lever le voile sur les mystères de la génération. La gestation constituait en effet par tradition un point fort dans les musées anatomiques : la Vénus de Susini abritait un fotus discret; et la première cire anatomique, réalisée par Desnoues et Zumbo au tout début du XVIII siècle à Gènes avait été moulée d'après nature sur une femme enceinte.

La section accouchement, qui contenait des coupes de bassins de femmes grosses, était suivie d'une importante section d'embryologie, qui permettait de pénétrer - dans une démarche anatomique - plus avant encore dans l'intérieur du corps enceint, c'est-àdire plus loin dans les secrets de la procréation et de la vie. Le spectateur pouvait alors contempler dans des vitrines des embryons et des fœetus à plusieurs stades, à l'état de squelettes, de cires, de pièces injectées, ainsi que des coupes d'utérus. La richesse de ces sections valorisait fortement la fonction reproductrice de la femme.

Les quatre cires consacrées à l'accouchement étaient sans doute les plus impressionnantes, car elles étaient réalisées avec le même soin que les Vénus, dont elles constituaient d'une certaine façon des déclinaisons : grandeur nature, et avec des implantations pileuses naturelles. Un seul des quatre mannequins présentait un " accouchement naturel ", avec la tête et les épaules d'un enfant surgissant entre les cuisses à peine entrouvertes d'une femme en chemise, au sexe invisible. Les trois autres donnaient à voir des parturitions chirurgicalement assistées, au crochet, au forceps ou par césarienne, dont les indications et le déroulement étaient soigneusement décrits dans les notices des catalogues. La comparaison entre les catalogues montre que le texte sur la césarienne a subi des transformations significatives entre la fin du $\mathrm{XIX}^{\mathrm{e}}$ siècle et le milieu du $\mathrm{xx}^{\mathrm{e}}$ siècle, au fur et à mesure que l'opération s'est sécurisée et 
banalisée grâce à l'asepsie. Lorsque la pièce rejoint la collection Spitzner, au XIx ${ }^{\mathrm{e}}$ siècle, la césarienne est toutefois encore une opération à haut risque en raison des dangers d'hémorragie, de péritonite et de septicémie inhérents à toute chirurgie abdominale. Il s'agit en outre d'une césarienne traditionnelle, in situ, c'est-à-dire où la matrice est ouverte en place (et non extériorisée avant d'être incisée, comme on commence à le faire au début du xxe siècle).

17 C'est peut-être la raison pour laquelle la représentation en paraît si dramatique, surtout comparée aux trois autres. Car l'expression du visage, la bouche entrouverte, tournée vers la plaie béante, n'est pas sans rappeler celle de la frayeur recréée par Duchenne de Boulogne dans ses recherches sur le Mécanisme de la physionomie humaine (1862). La façon dont les pieds sont attachés et les bras maintenus derrière la tête évoque les planches (cruelles) de Gérard de Lairesse pour l'Anatomie de Bidloo (1685), ou la Vénus anatomique de Chauvet, exhibée à Londres au XviII ${ }^{\mathrm{e}}$ siècle, qui montrait la circulation sanguine chez une femme enceinte mise en scène comme si elle était victime d'une dissection in vivo ${ }^{22}$. Si ces références avaient peu de chances d'être connues du public, la proximité spatiale de cette cire avec de nombreux moulages issus de dissections pouvait peut-être, sans le secours de la notice, inciter à la voir comme une scène de vivisection. Les quatre mains, convergeant vers le ventre ouvert, opèrent une mainmise littérale sur le corps; et cette violence suggérée par la composition contraste d'autant plus fortement avec la chemise de nuit raffinée qui enveloppe la parturiente. Enfin, si comme Elisabeth Stephens le signale à juste titre le sexe de la femme n'est pas visible ${ }^{23}$, l'incision en mandorle le rappelle fortement; de sorte que l'ensemble suggère tout à la fois la vivisection, le crime et le viol.

Attention toutefois aux risques de surinterprétation : une lecture plus «technique » de la cire livre d'autres hypothèses: les bras sont relevés pour dégager le champ opératoire, les jambes liées pour éviter peut-être tout mouvement qui pourrait faire saillir les intestins hors de l'abdomen; les mains représentées sont affectées à des tâches bien précises, qui témoignent des efforts de la chirurgie pour se moderniser : éponger le champ opératoire, pousser l'utérus vers la droite pour l'aligner sur l'axe de la ligne blanche, inciser avec le bistouri. Reste l'interprétation de l'expression du visage d'autant plus énigmatique que l'anesthésie, devenue systématique depuis le milieu du siècle, devrait plonger la patiente dans le sommeil... Sauf à supposer que la scène représente une césarienne post mortem ? Une hypothèse que n'interdit pas la notice, qui indique que «le plus souvent, cette opération se pratique la mère étant morte et l'enfant encore vivant $»^{24}$. 
Fig. 4

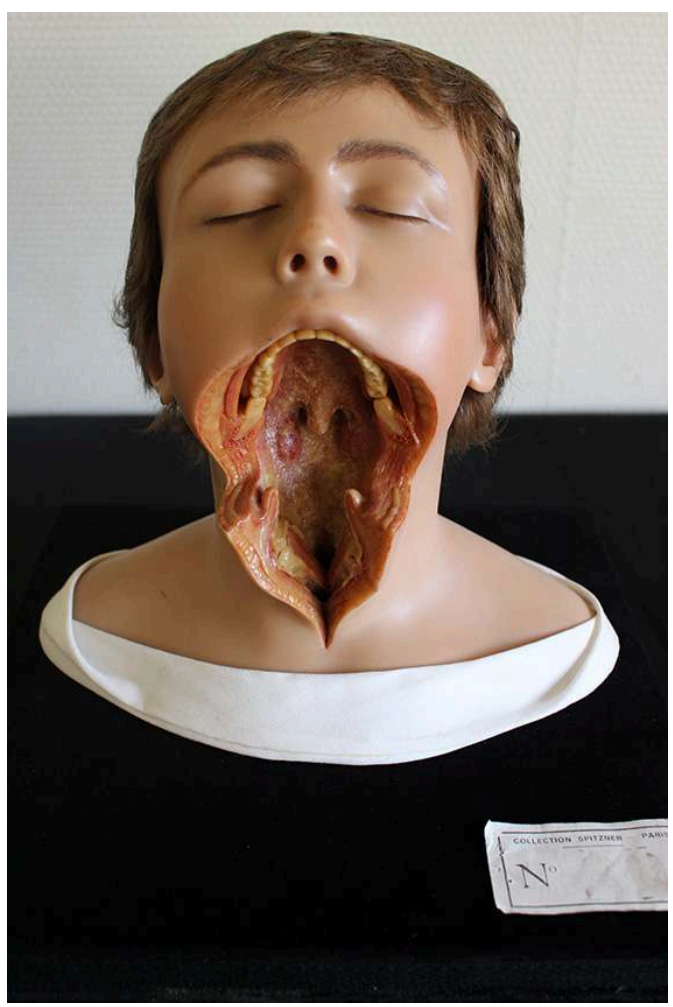

Diphtérie. xıx siècle. Cire colorée, cheveux naturels, yeux en verre soufflé. Collection Pierre Spitzner. Classement en 2004 au titre des monuments historiques. Faculté de médecine de l'Université de Montpellier (Inv. UM.ANAT.DOR. 1311).

(C) Université de Montpellier

19 Le musée présentait de nombreuses cires d'anatomie pathologique » moulées sur nature », qui allaient de maladies dermatologiques à des lésions viscérales en passant par des fractures ou des tumeurs. Certaines pathologies étaient particulièrement mises en valeur, sans doute en fonction de leur fréquence, comme les «affections de l'utérus ", des yeux, ou la tuberculose. Pour inciter le public à la prudence, une vitrine en montre à l'extérieur exposait «à l'œil nu » dans un bassin grossi, "les trichines exerçant leurs ravages »: il s'agissait de parasites intestinaux associés à la consommation de viande de porc mal cuite. Une section entière, enfin était affectée à la diphtérie. Cette maladie, parfois appelée croup, était la terreur des mères dont elle frappait surtout les enfants entre 2 et 7 ans, et une préoccupation de santé publique pour les gouvernements dans un contexte de faible natalité. Elle se marquait par une toux rauque, puis un étouffement menant à la mort par l'obstruction progressive du larynx par congestion des membranes. Par sa létalité, son caractère fulgurant, le supplice qu'elle infligeait et sa prévalence, le croup occupait une place très importante dans les imaginaires $d u \mathrm{XIX}^{\mathrm{e}}$ siècle, à travers les romans feuilletons, la littérature (Brienne, Gozlan, Toudouze, Flaubert) ou la poésie (Daudet, Hugo). Une série de trois cires illustrait donc les effets du croup sur un garçon de $6 \mathrm{ans}^{25}$. La première montrait la gorge à l'état sain, la deuxième les premiers symptômes, et la troisième le "plein développement de la maladie ». Pour rendre visible les symptômes, la tête était privée de mâchoire inférieure, dans une configuration sans doute impressionnante pour les visiteurs. Le livret-catalogue jouait ici pleinement son rôle pédagogique auprès des parents, comme le montre la notice qui accompagnait la deuxième cire : "L'enfant a 
une légère fièvre, un sommeil interrompu, une humeur moussante, la voix devient rauque et la visite du médecin est urgente $»^{26}$.

Le croup est resté longtemps sans autre remède que la trachéotomie, c'est-à-dire une incision verticale du cou puis de la trachée, et la mise en place d'une canule pour permettre à l'air de circuler à nouveau dans les voies respiratoires. Bien que cette opération soit citée comme «la seule ressource» par le catalogue, aucune cire ne la représente avant le $\mathrm{xx}^{\mathrm{e}}$ siècle, ce qui pose la question de la place de la chirurgie dans les collections. En 1895, celle-ci avait pourtant connu un développement sans précédent grâce à la mise au point de l'anesthésie, des pinces hémostatiques et de l'antisepsie. De fait, quelques cires représentaient la ligature des artères ou les opérations de la cataracte, de la césarienne et de la lithotritie, mais il s'agissait à vrai dire de gestes pratiqués depuis longtemps et dont le succès n'était pas toujours garanti. Le musée Spitzner semble donc avoir privilégié la mise en spectacle du progrès médical par rapport au progrès chirurgical : les visiteurs pouvaient admirer des scènes grandeur nature représentant "La transfusion du sang", "Pasteur et la rage », "Charcot et l'hystérie »; dans ce dernier cas, la scène était la représentation en trois dimensions du tableau d'André Brouillet, Une leçon clinique à la Salpetrière (1887) ${ }^{27}$.

Dans le cas du croup, cette confiance dans la médecine se marque par le choix de représenter parmi ces scènes reconstituées «Le docteur Roux dans son laboratoire ". $\mathrm{Ce}$ collaborateur de Pasteur venait en effet de mettre au point un sérum antidiphtérique qui allait permettre l'éradication de «ce monstre exterminateur de l'enfance ». Avec Pasteur et Roux, le musée Spitzner faisait donc adhérer ses visiteurs à la foi en la microbiologie naissante et en ses bienfaits pédiatriques.

Fig. 5

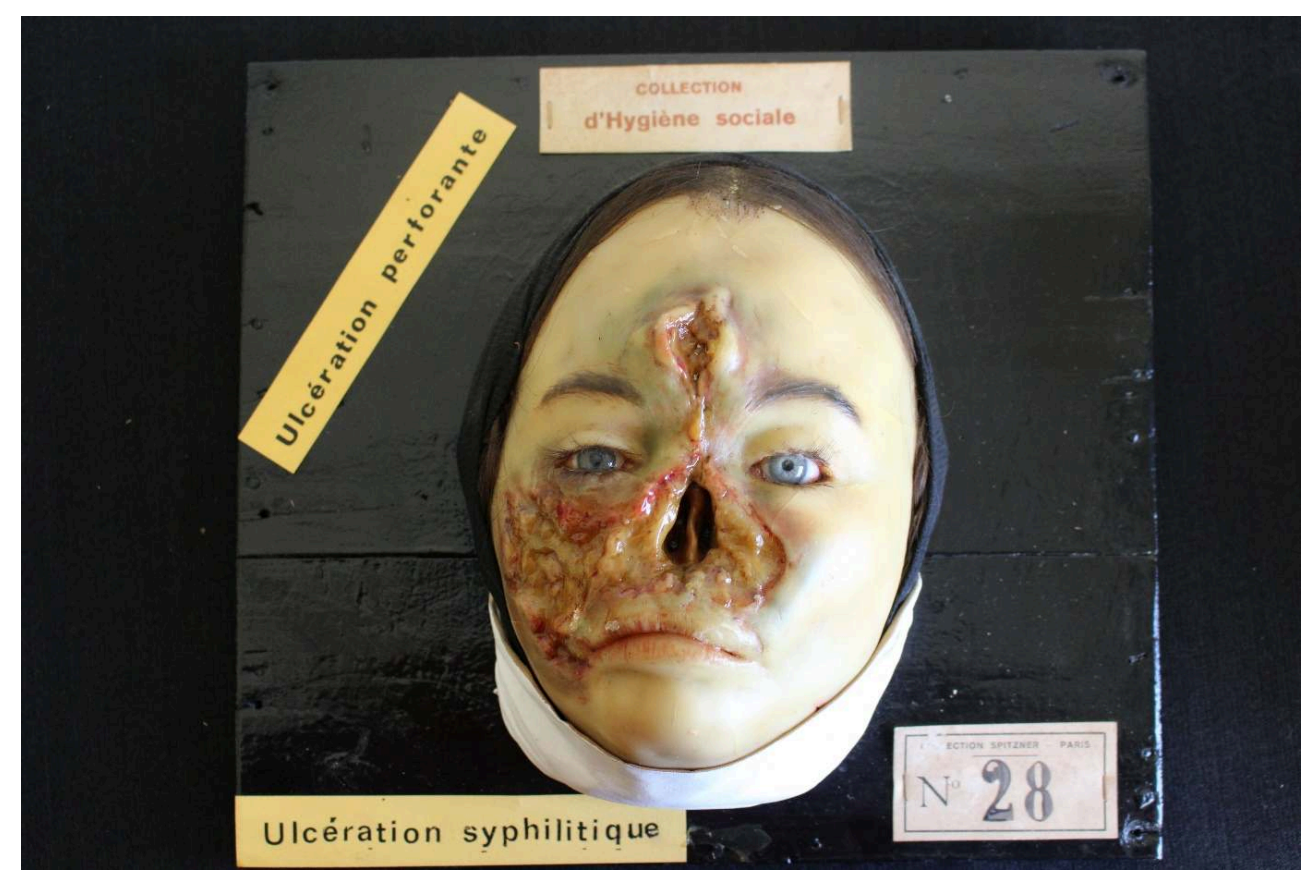

Syphilis ulcéreuse et perforante. xix ${ }^{\mathrm{e}}$ siècle. Cire colorée, cheveux naturels, yeux en verre soufflé. Collection Pierre Spitzner. Classement en 2004 au titre des monuments historiques. Faculté de médecine de l'Université de Montpellier (Inv. UM.ANAT.DOR. 1419).

(c) Université de Montpellier 
Elles s'inscrivaient dans le prolongement du musée de cires Bertrand-Rival, actif au début du xix ${ }^{e}$ siècle, qui montrait dans quelques vitrines les ravages de la masturbation sur le visage, le corps et les organes génitaux des malheureux qui s'y adonnaient ${ }^{30}$. Bien que le musée Spitzner présentât quelques pièces faisant mention des effets de l'onanisme, l'urgence était plutôt alors à dissuader les spectateurs de recourir à la sexualité mercenaire, désignée comme le vivier du péril vénérien. C'est la raison pour laquelle les militaires, public cible de ce type d'exposition, avaient droit à un demi-tarif. Une gravure de Félicien Rops montre un jeune conscrit tétanisé devant une vitrine, que lui commente un supérieur ${ }^{31}$.

Ces cabinets réservés firent progressivement l'objet de critiques des moralistes, qui les accusaient de pornographie - à l'instar des néomalthusiens et de leurs brochures illustrées; et cette accusation contribua à contaminer l'ensemble des musées anatomiques, accusés de flatter les goûts dépravés de la foule. Le filtrage du public et l'utilisation de moulages provenant de la même main et en tous points identiques à ceux présentés dans les vitrines hospitalo-universitaires constituaient pourtant des garanties morales réelles. Chloé Pirson remarque à juste titre que ce type de cabinet réservé était le seul espace de pédagogie populaire où l'on pouvait réellement prendre conscience des effets de la syphilis ; qu'il assurait, à ce titre, les missions dévolues peu après aux musées d'hygiène sociale, et recevait les mêmes visiteurs institutionnels : armée, sociétés médicales, écoles d'infirmières, etc. comme en atteste la correspondance de la veuve de Pierre Spitzner. Ce mépris des foules, cette condamnation morale et cette pulsion censoriale ne sont pas sans rappeler celles qui 
visent, au même moment, la visite populaire à la morgue, pourtant instituée dans un but médico-légal d'identification ; ou le spectacle public de l'exécution capitale, justifié au départ par son exemplarité et ses vertus édifiantes ${ }^{32}$.

Fig. 6

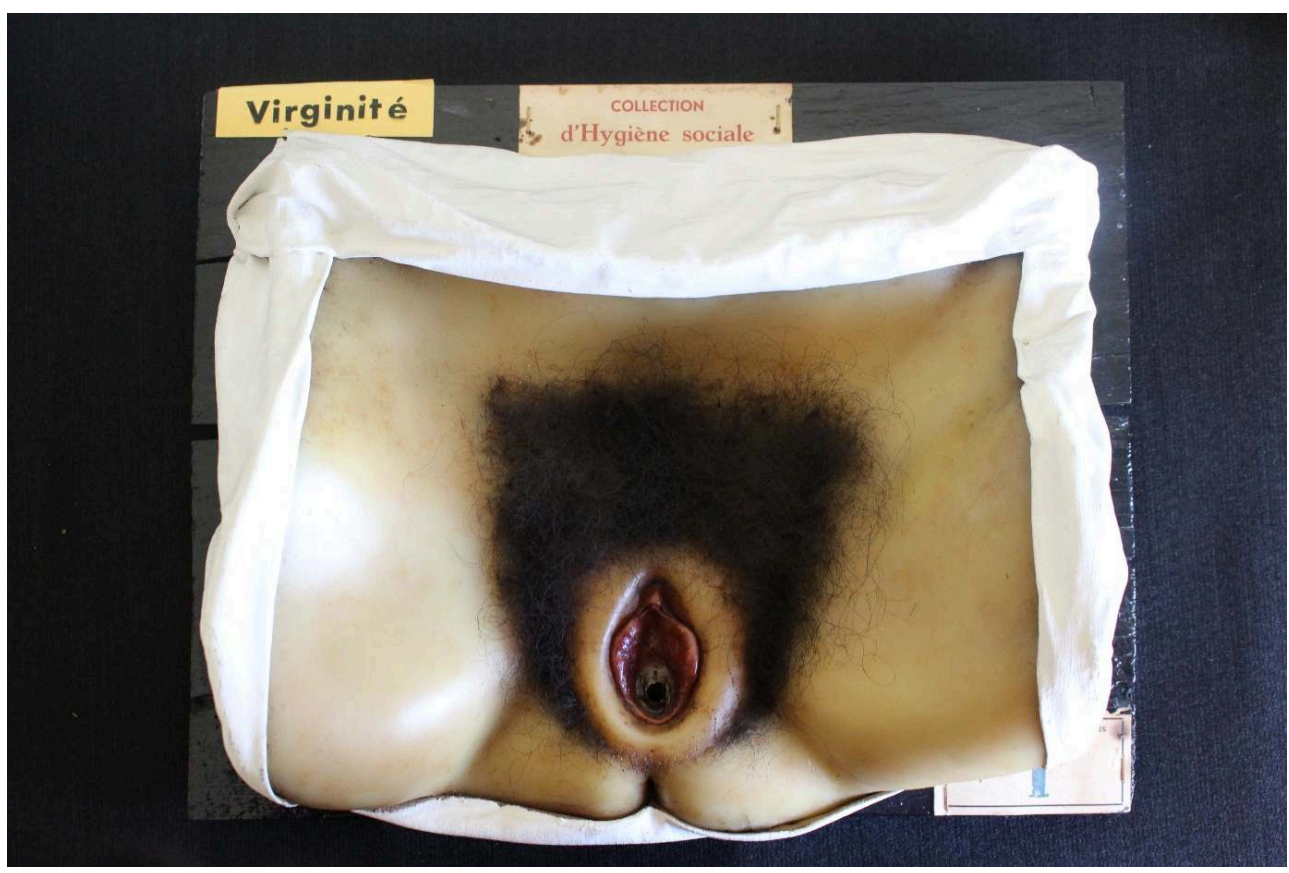

Vagin, partie sexuelle à l'état vierge. xıx siècle. Cire colorée, poils naturels. Collection Pierre Spitzner Classement en 2004 au titre des monuments historiques. Faculté de médecine de l'Université de Montpellier (Inv. UM.ANAT.DOR. 1243)

(C) Université de Montpellier 
Fig. 7

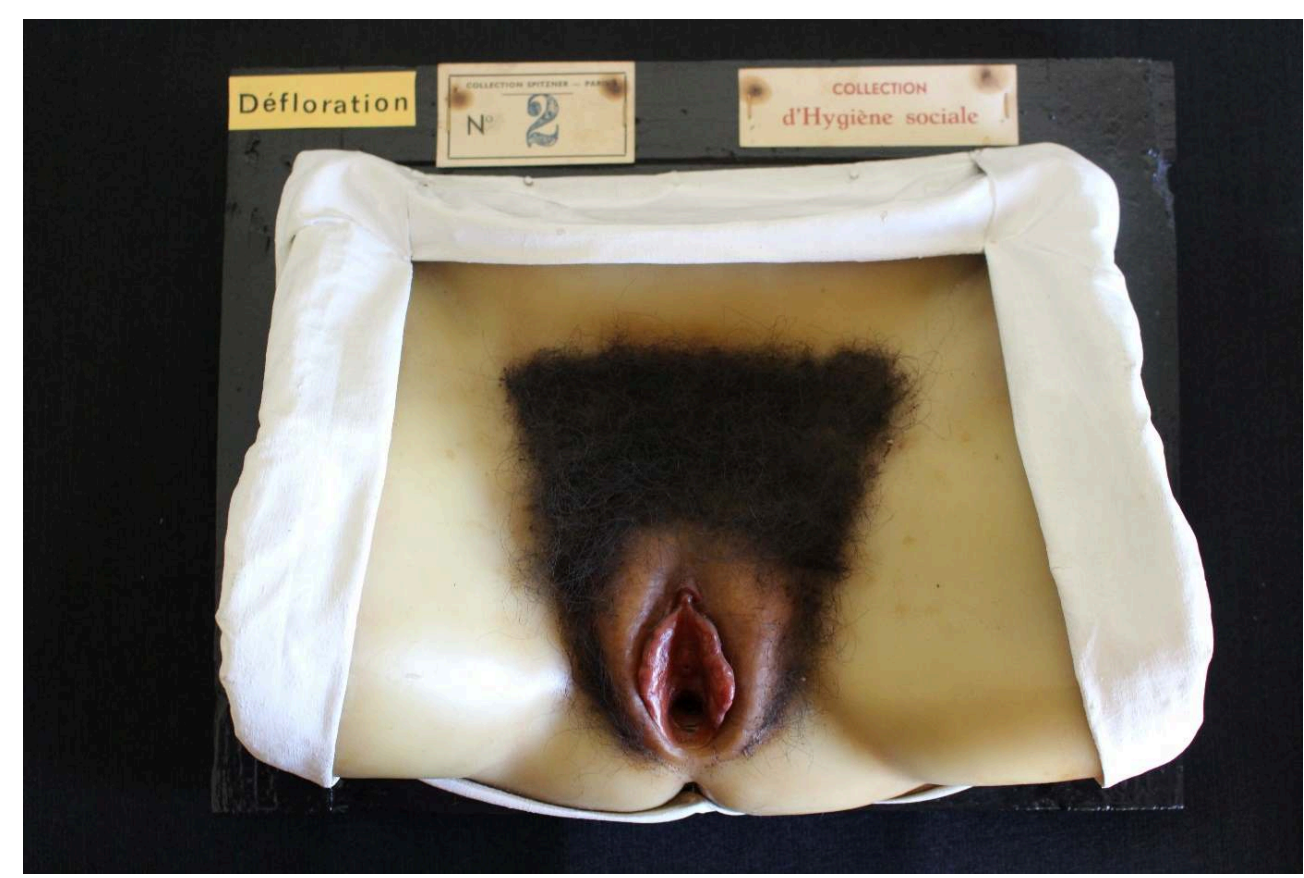

Vagin, partie sexuelle après défloraison. $x x^{\mathrm{e}}$ siècle. Cire colorée, poils naturels. Collection Pierre Spitzner. Classement en 2004 au titre des monuments historiques. Faculté de médecine de l'Université de Montpellier (Inv. UM.ANAT.DOR. 1244)

(C) Université de Montpellier

Dans la galerie réservée, les pièces présentées avaient des cadrages plus resserrés sur les parties de corps (face, mains, seins, etc., et surtout organes sexuels) atteints par la maladie. La céroplastie qui avait accompagné au début du xix ${ }^{e}$ siècle la montée de la méthode anatomo-clinique s'était en effet détournée en France de ses mises en scènes baroques pour se concentrer sur l'anatomie pathologique, et sur le rendu le plus réaliste possible des lésions. Les moulages effectués par Baretta notamment s'inscrivaient donc dans un cadre neutre, focalisé sur l'organe atteint; la focalisation était accentuée par l'entourage de la zone représentée par un linge blanc, rappelant les contours du champ opératoire en chirurgie; les visages étaient traités, hors du périmètre de la lésion, de façon peu expressive. En dépit de cette présentation "froide », la galerie réservée créait toutefois un effet d'accumulation fascinant. Elle offrait aux yeux un alignement serré de hauts et bas-reliefs d'échelle et d'agencement identiques, contre lesquels le regard n'en finissait pas de rebondir, comme autant de variations de tumeurs proliférantes, de sexes aberrants et de visage dévastés.

Dans le cas de cette double cire, qui ouvrait la galerie, la dimension pathologique est absente. Le choc visuel provient du face-à-face entre le spectateur et le sexe largement ouvert, qui renvoie immédiatement aux clichés pornographiques qui commencent à se répandre sous le second Empire ${ }^{33}$. La contemplation frontale et rapprochée du sexe féminin gardait un caractère puissant de crudité, y compris dans la pratique médicale ; ainsi, la diffusion du spéculum gynécologique, au milieu du xix siècle, rencontre de grandes réticences chez certains médecins, entre autres parce qu'il semblait heurter bien plus la pudeur que le toucher, pratiqué à l'aveugle sous les jupes des patientes ${ }^{34}$.

On peut s'interroger sur la raison d'être d'un tel moulage dans les collections du musée. 
existence pourrait attester de la place persistante, dans les préoccupations des contemporains, de la virginité féminine. On sait pourtant que les médecins s'attachent au cours du siècle à combattre les préjugés en la matière et notamment le surinvestissement de la membrane hymen, comme seul signe de virginité. Les mêmes plaident pour que la douceur prévale dans les premiers rapports sexuels. La seconde cire, qui montre les effets de la défloration (tuméfaction, allongement des lèvres, etc.) va peut-être dans ce sens. Reste que la figuration précise de l'hymen dans la première cire a paradoxalement sans doute eu pour effet de conforter sa fiabilité comme signe de virginités ${ }^{35}$.

En réalisant L'Origine du Monde en 1866, Gustave Courbet peint un fragment corporel d'un corps féminin, le sexe. Or, l'artiste fréquentait les cabinets de cire comme le raconte une nouvelle de Champfleury parue en $1849^{36}$. La peinture de Gustave Courbet s'inspire des codes des musées anatomiques : cadrage resserré sur une partie du corps qui ne permet pas d'identifier le sujet. D'ailleurs, cette parenté avec les collections anatomiques est perçue à l'époque, comme le souligne un critique anonyme en 1870, qui affirme que « cette chose eût été la bienvenue dans ce musée amoroso-chirurgical du boulevard Bonne-Nouvelle, où l'on ne laissait entrer ni les femmes ni les hommes en dessous de vingt ans $»^{37}$.

Fig. 8

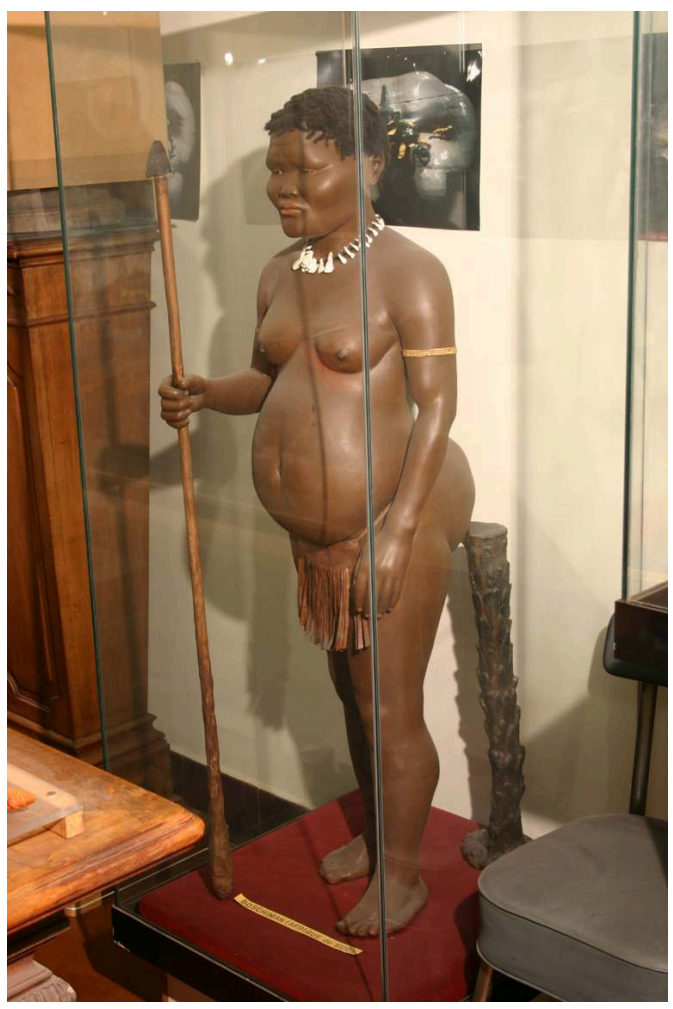

Vénus Hottentote. xix siècle. Cire colorée, cheveux naturels, yeux en verre soufflé. Collection Pierre Spitzner. Classement en 2004 au titre des monuments historiques. Faculté de médecine de I'Université de Montpellier (Inv. UM.ANAT.DOR. 1457)

(C) Université de Montpellier

Outre sa collection d'anatomie normale et pathologique, le Musée Spitzner présentait une petite collection ethno-anthropologique d'une douzaine de pièces, censées présenter les caractéristiques raciales des variétés de l'humanité. Le catalogue de 1895 
mentionne des bustes d'Arméniens, d'Abyssiniens, de Cafres, de Samoyèdes, d'Australiens, d'Aztèques, etc. La taille modeste et le faible développement ultérieur de ces collections s'expliquent peut-être par la concurrence des zoos humains contemporains, et plus localement, du Palais des colonies de Tervueren, construit dans le cadre de l'Exposition universelle de Bruxelles de 1897, qui abritait plus de 300 Congolais dans des villages reconstitués ${ }^{38}$. Ces pièces étaient accompagnées dans le catalogue de notices expédiant en quelques lignes caricaturales les caractéristiques physiques, intellectuelles et morales des « races » représentées, renvoyant les acquis de l'anthropologie de terrain plusieurs dizaines d'années en arrière ${ }^{39}$.

32 La Vénus hottentote était présentée à part, dans la dernière section du musée, sous la forme d'une cire en ronde-bosse et en pied $(1,45 \mathrm{~m})$, censée avoir été moulée d'après nature. La "Vénus hottentote» originelle, Saartjie Baartman, avait été ramenée d'Afrique australe en 1810 pour être exhibée dans des spectacles dégradants en Angleterre au motif de sa stéatopygie et de sa macronymphie ${ }^{40}$ (le « tablier hottentot »), considérés alors comme des éléments spécifiques à sa "race ", et qui en faisaient à la fois un objet exotique, pornographique et un phénomène de foire. C'est à l'occasion de ces exhibitions qu'on lui avait donné par dérision le surnom de "Vénus noire » ou « hottentote ». Elle avait fini par mourir à Paris en 1815, non sans avoir fait l'objet auparavant d'une série d'examens par Cuvier au Museum et par Etienne Geoffroy SaintHilaire à l'Académie des Sciences, d'un moulage puis d'une dissection.

La notice qui accompagne la cire du musée Spitzner est un condensé de stéréotypes et d'erreurs :

Cette femme, de son vivant, fut exhibée en Angleterre, en Danemark et en Allemagne. Elle mourut à Ulm en 1806. Les Boschimans sont les nègres les plus remarquables par leur constitution ; ils sont très paresseux et restent couchés sous les buissons pendant plusieurs jours, jusqu'à ce que la faim les pousse à la recherche de leur nourriture. Ils sortent clandestinement pour enlever les brebis dans les troupeaux. (Cette femme est modelée sur nature. On la voit également au Muséum d'histoire naturelle, à Paris). ${ }^{41}$

La cire ne ressemble absolument pas, en effet, au moulage en plâtre conservé par le Museum; ni les traits du visage, ni la poitrine, ni la silhouette, ni la posture ne sont identiques. En réalité, le terme de "Vénus hottentote » est devenu générique et a servi à désigner de nombreuses femmes Khoisans « importées » et exhibées pour satisfaire la curiosité populaire et scientifique, attisée par la première Vénus. L'une d'elles, Afandy, est morte à Ulm, en 1866, alors qu'elle effectuait une tournée dans le pays, et son corps a été confié à un professeur d'anatomie, Luschka ${ }^{42}$. On peut faire l'hypothèse que c'est un moulage d'Afandy qui figure dans les collections Spitzner. Reste à déterminer si les erreurs dans la notice, qui tendent à faire croire au public qu'il se trouve en face de la « première » Vénus, sont ou non délibérées, dans le but d'attirer le curieux.

Quoi qu'il en soit, la contemplation de cette Vénus ne pouvait manquer de faire un écho ironique aux deux autres Vénus présentées dans le musée, lesquelles correspondaient parfaitement aux canons esthétiques du temps. La dissimulation de ses soi-disant spécificités anatomiques, d'ailleurs absentes de la notice, semble indiquer toutefois qu'il ne s'agit là que d'une pièce à prétention ethnologique; vêtue d'un pagne, ornée d'un collier et d'un bracelet autour du bras droit, armée d'une lance, elle rappelle les « indigènes » de fantaisie dessinés par Jules Chéret sur l'affiche qu'il réalise en 1897 pour le musée. 
Fig. 9

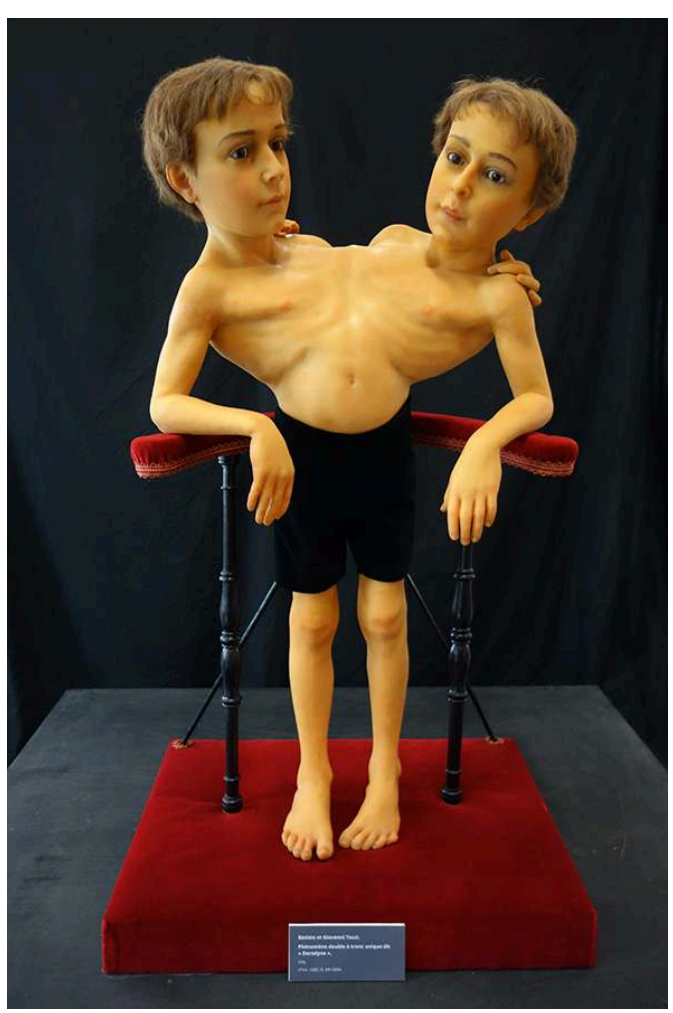

Frères Tocci. xix ${ }^{\mathrm{e}}$ siècle. Cire colorée, textile, cheveux naturels, yeux en verre soufflé. Collection Pierre Spitzner. Classement en 2004 au titre des monuments historiques. Faculté de médecine de I'Université de Montpellier (Inv. UM.ANAT.DOR. 1283)

(c) Université de Montpellier

Les spectacles de phénomènes vivants connaissent un grand succès sur les foires dans la seconde moitié du XIX ${ }^{\mathrm{e}}$ siècle. C'est à partir des années 1850 que se développent les baraquements forains et les grandes entreprises du show business, qui connaissent leur apogée dans les années 1880 ; la différence, l'étrangeté, la difformité et l'infirmité y étaient mises en scène sur des modes terrifiants ou comiques, rarement pédagogiques ${ }^{43}$. Les spectateurs venaient observer ces corps pour se procurer des sensations fortes, inhabituelles et sidérantes, se divertir, et peut-être conforter leur propre sentiment de normalitét ${ }^{44}$.

37 Le musée Spitzner présentait une reproduction en cire des frères Tocci (Giacomo et Giovanni, nés en 1877) grandeur nature, âgés d'une dizaine d'années. Ces deux frères possédaient une paire de jambes et un abdomen communs, pour deux thorax, deux paires de bras et deux têtes. Il s'agissait d'un exemple de "monstre en $Y$ », double en haut et simple en bas, ou pour reprendre la taxinomie savante inventée par Isidore Geoffroy Saint-Hilaire au début du siècle, d'un xiphodyme. Dans les années 1880, les frères Tocci avaient été exhibés in vivo en Europe puis aux États Unis dans les années 1890 ; des photographies d'eux circulaient également à la même époque. Au sein du musée, c'est la seule figure de monstre présentée ainsi, quelques malformations bénignes comme le bec de lièvre étant dispersées dans le reste des collections ${ }^{45}$.

Pour autant, le musée Spitzner a aussi exhibé des phénomènes vivants, en profitant de leur tournée en Europe. En février-mars 1873, il avait présenté à Marseille les sœurs Millie-Christine (un moulage de leur bassin et de ses particularités était visible dans 
une vitrine), deux jeunes filles nées en 1851 en Caroline du Nord et soudées par le sacrum, que le cirque de Barnum exposait dans un numéro musical sous le nom de «Rossignol à deux têtes ». En 1897 et 1898, le musée accueille les sœurs indiennes Radica et Doodica, nées soudées par le thorax en $1888^{46}$. Il participe donc de fait à la mise en spectacle des anormalités physiques, au plus fort de son succès populaire.

Pourquoi une telle cire, et de telles exhibitions? Sans nier leur pouvoir d'attraction sur des publics aux motivations variées et la probabilité que Spitzner y ait été sensible, il ne faut pas oublier que les phénomènes anatomiques constituaient des pièces de choix dans les Wunderkammer et autres cabinets de curiosité, dont les musées anatomiques reprenaient en partie la tradition. Contrairement aux monstres par défaut, les monstres doubles étaient traditionnellement de "beaux monstres "; au XVIII ${ }^{\mathrm{e}}$ siècle, leur symétrie parfaite ne pouvait être accidentelle aux yeux de certains savants et elle attestait de la liberté et de la toute-puissance de la création divine ${ }^{47}$. Au XIX ${ }^{e}$ siècle, et notamment depuis la tournée des frères siamois Chang et Eng, ils faisaient l'objet de la curiosité avide des savants tout autant que de celle des spectateurs, en même temps que la tératologie se constituait en science ${ }^{48}$. Leurs employeurs se prêtaient d'autant plus volontiers à l'investigation médicale qu'une expertise authentifiait et valorisait le caractère exceptionnel de leur attraction.

La présence des frères Tocci dans les collections Spitzner ne doit donc pas tant être interprétée comme une dérive du musée vers le freakshow que comme un moyen de faire percevoir, dans la continuité des vitrines d'embryologie, les lois mystérieuses de développement du vivant. On peut dès lors s'interroger sur les conditions de production de cette cire: s'agit-il d'une cire moulée sur nature, lors de la tournée française des Tocci en 1883 ou d'une création d'après photographie? La figure emprunte en partie la " pose », les accessoires (les accoudoirs de fauteuil remplacés par une rampe capitonnée) aux séries de portraits des frères réalisées dans l'atelier d'un photographe, qui circulent au même moment. Mais le visage des frères Tocci est particulièrement soigné (cheveux, cils et sourcils naturels), et comparées à ces images, leurs jambes sont redressées, leur dissymétrie est atténuée, leur nudité voilée, leur fraternité mise en scène de façon touchante ; le réalisme est quelque peu malmené, au profit d'une forme d'idéalisation, plus propre à susciter l'admiration que la répugnance. C'est sans doute à ce prix que les frères Tocci peuvent être présentés au public, selon le catalogue, comme "incontestablement le phénomène le plus curieux qu'on ait pu voir $»^{49}$. 


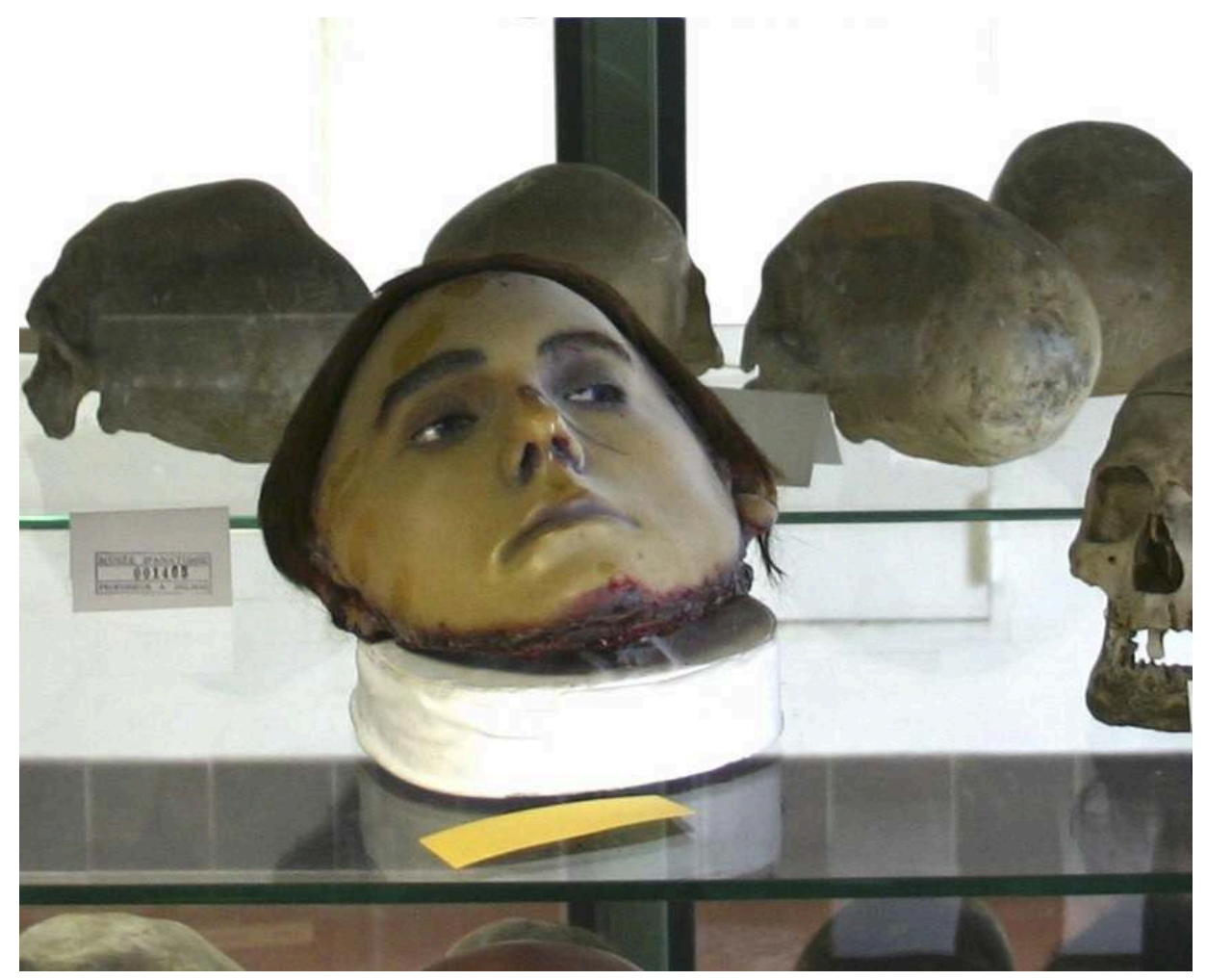

Tête de décapité de Vannieuwenhove. xix ${ }^{\mathrm{e}}$ siècle. Cire colorée, cheveux. Collection Pierre Spitzner. Classement en 2004 au titre des monuments historiques. Faculté de médecine de l'Université de Montpellier (Inv. UM.ANAT.DOR. 1465)

(C) Université de Montpellier

41 Le musée présentait à la fin du xix ${ }^{e}$ siècle une vitrine de squelettes et de crânes contenant quelques têtes, dont la "tête d'un Arabe exécuté ». À cette collection un peu disparate s'ajoutait, hors section, le moulage de la tête d'un criminel, Emile Vannieuwenhove, guillotiné le 21 avril 1894 pour un triple meurtre particulièrement sauvage. Plus tôt, en 1888, le musée s'était adjoint temporairement un moulage de la tête de Pranzini, guillotiné en 1887.

La présence de cette tête dans les collections s'inscrit dans le contexte de trois curiosités confluentes. La première, savante, était celle portée par l'anthropologie criminelle qui s'était développée au xIX ${ }^{e}$ siècle dans le sillage de la phrénologie de Gall. De vastes collections de crânes, de cerveaux, de têtes prélevées ou moulées après l'exécution de criminels avaient été constituées dans les laboratoires d'anthropologie, les collections des facultés et le Museum. Ces collections offraient un terrain d'études à ceux qui, comme Lombroso en Italie ou Dumoutier en France, traquaient les stigmates des penchants criminels dans la physionomie ou la topographie des circonvolutions cérébrales ${ }^{50}$. D'autres médecins s'intéressaient aux têtes fraîchement coupées dans les années 1880 : ceux qui doutaient de l'instantanéité de la mort par décapitation, et souhaitaient soit s'en assurer par des expériences, soit tenter des revivifications ${ }^{51}$.

La seconde forme de curiosité, plus commune, participait de la médiatisation contemporaine du crime. Le développement de la presse populaire dans le dernier tiers du siècle s'était appuyé notamment sur le goût pour le sensationnel et le fait-divers violent. La description des circonstances du crime, le récit de l'enquête policière et de 
l'arrestation, la restitution du procès, puis la narration de l'exécution du coupable fournissaient la matière d'un feuilleton journalistique qui tenait les lecteurs en haleine pendant des semaines ${ }^{52}$. Ce goût pour le crime était exploité au même moment par d'autres types de spectacles : au théâtre (du théâtre de boulevard au Grand Guignol, ouvert en 1897) ou dans des attractions comme le célèbre musée Grévin, ouvert en 1882. À l'instar du musée Spitzner, celui-ci exposait des cires grandeur nature ; il offrait à ses très nombreux visiteurs une sorte de représentation en trois dimensions et en action de ceux qui avaient fait l'histoire, mais aussi et surtout de ceux qui faisaient l'actualité des journaux, comme les célébrités du Tout-Paris ou les protagonistes des grandes affaires criminelles ${ }^{53}$. Le musée Grévin offrait ainsi une série de sept tableaux consacrés à "L'histoire d'un crime », de sa perpétration jusqu'à la toilette du condamné à mort dans la prison.

Enfin, le point final fréquent des affaires criminelles, l'exécution, restait à la fin du siècle un spectacle couru des foules. Pourtant, toute une série de mesures avaient été adoptées pour en minimiser l'éclat. L'heure des exécutions avait été fixée de plus en plus tôt dans la journée; celles-ci se déroulaient de plus en plus près des prisons et depuis 1871 , l'échafaud avait été supprimé, mettant la machine au niveau du sol, alors que la foule était contenue à distance par un cordon de troupe. Le spectacle était donc en réalité quasiment invisible pour la plupart des spectateurs, et le corps du condamné rapidement escamoté par les exécuteurs.

Le choix de présenter le moulage d'une tête de guillotiné pouvait donc se justifier par la curiosité scientifique ou la pulsion scopique des amateurs de récits criminels. En l'occurrence, le musée joue surtout la seconde carte : la tête rejoint opportunément les collections au moment même où le musée se déplace dans le nord de la France et la Belgique, précisément là où Vannieuwenhove a grandi (Bruxelles), accompli son forfait (Leers, près de Douai), été exécuté (Lille). Le moulage de sa tête avait été réalisé une heure après l'exécution à la faculté de médecine de Lille où le corps avait été emporté pour être disséqué par le docteur Debierre - professeur d'anatomie - et faire l'objet d'expériences par le médecin légiste Castiaux ${ }^{54}$. L'objet, d'origine scientifique, se transforme donc en intégrant les collections en une attraction sensationnelle.

Spitzner avait sans doute conscience de cette ambiguïté : à la fois du potentiel fascinant de ce type de cire, et de son caractère déplacé dans son musée. En 1888, après un incendie qui l'avait obligé à rénover ses collections, peut-être stimulé par le succès de Grévin, il avait intégré au musée des pièces historiques (le général Boulanger) et criminelles (l'assassin Pranzini, bientôt rejoint par d'autres figures d'affaires célèbres). Mais ces pièces n'apparaissent plus dans le catalogue de 1895 où la tête de Vannieuwenhove reste isolée dans son genre ; elles en ont été retirées. Quelques années plus tard, le fils de Spitzner prend la direction d'un deuxième lieu d'exhibition, le Musée historique, doté d'un catalogue distinct ${ }^{55}$. Celui-ci présentait une riche galerie de meurtriers et de victimes célèbres incluant Fieschi, Troppmann, Ravachol ou Jack l'Éventreur, auxquels s'ajoutaient quelques monstres, quelques grandes figures d'actualité comme Zola ou Dreyfus, ainsi qu'une série terrifiante d'instruments de torture de l'Inquisition : c'était clairement le frisson qui était recherché. 


\section{Conclusion}

Si le parcours que nous venons de proposer dans le musée Spitzner en 1895 est - nous l'espérons - représentatif, il n'est pas exhaustif. Le visiteur pouvait y contempler d'autres pièces, dont l'inventaire est inévitablement étrange et poétique: s'y rencontraient au hasard des bocaux ou des vitrines par exemple une «tête de fotus coupée en deux ", une "main gauche d'un singe dont l'épiderme est enlevé sur trois doigts ", "le larynx et la thyroïde d'une jeune femme ", le "squelette d'un tambour major, sortant du génie, mort à l'hôpital de Montpellier", un "homme naturel injecté ", un "chef de tribu indien", une "momie égyptienne", "deux peaux humaines tannées" ou "Jeanne d'Arc ». Ces pièces parfois un peu marginales inscrivaient le musée Spitzner dans des dynamiques contradictoires, des cabinets de curiosité obsolètes aux dioramas historiques à la mode, et menaçaient d'en brouiller légèrement la cohérence. Dans l'entre-deux-guerres, le musée oscille entre deux vocations : une vocation pédagogique (il est reconnu d'utilité publique par l'État belge en 1926, et est utilisé pour former des soignants) et une vocation distrayante, liée à son étrangeté croissante. Mais ces deux stratégies sont menacées. La pédagogie anatomique et médicale du musée commence à être concurrencée par d'autres media : la médecine avait changé d'échelle et traquait l'infiniment petit ; la photographie couleur suppléait aux cires, encombrantes et fragiles. Et qui pour s'émerveiller des squelettes à l'âge des rayons $\mathrm{X}$, eux-mêmes mis en scène dans les foires ${ }^{56}$ ?

\section{NOTES}

1. La guerre de 1870 contraint Pierre Spitzner à fuir Paris pour se réfugier à Marseille. En 1871, il commence à sillonner les foires d'Europe du Nord avec sa collection, tout en maintenant un ancrage parisien, où il expose dans différents locaux. Ainsi, il se rend dans le nord de la France (Lille, Roubaix, Nantes, etc.), mais aussi en Belgique (Gand, Bruxelles, Liège, Anvers, etc.), en Allemagne, aux Pays-Bas, en Angleterre. Il expose aussi deux fois aux États-Unis en 1875 et 1878.

2. La redécouverte du musée donne lieu à trois expositions (Ixelles en 1979, Paris en 1980 et Avignon en 1983). La collection est rachetée par Roussel-Uclaf en 1985 qui en fait don en 1997 à la Société française d'anatomie et de pathologie. Elle était exposée avec la collection Delmas-Orfila-Rouvière à Paris, et cédée à l'Université de Montpellier en 2011. (Voir : Hélène Palouzié et Caroline Ducourau, « De la collection Fontana à la collection Spitzner, l'aventure des cires anatomiques de Paris à Montpellier ", In Situ, $\mathrm{n}^{\circ}$ 31, 2017, en ligne : http://insitu.revues.org/14142 ; DOI : 10.4000/insitu.14142, p. 29 et note 53.)

3. Pierre Spitzner aurait été formé à l'art de la céroplastie par Paul Zeiller, un céroplaste travaillant pour des institutions allemandes.

4. Daniel Raichvarg, Science et spectacle. Figures d'une rencontre, Paris, Z'Éditions, 1993, p. 40.

5. Anonyme, «Le Museum », Le Progrès de la Côte-d'Or, n² 247, 4 septembre 1894, p. 2. 
6. Rafael Mandressi, Le Regard de l'anatomiste. Dissections et invention du corps en Occident, Paris, Seuil, 2003.

7. On pense notamment aux ouvrages éponymes publiés par Louis Figuier.

8. Anonyme, «Chronique locale. Roubaix. La Foire. Première promenade ", in L'Avenir de RoubaixTourcoing, $\mathrm{n}^{\circ}$ 7443, 23 avril 1895, p. 2.

9. Olivier Cousinou, et Laura Neve dir., Paul Delvaux, le rêveur éveillé, Gand, Snoeck, 2014, p. 59-63.

10. Chloé Pirson, Corps à Corps. Les modèles anatomiques entre art et médecine (1699-2008). Paris, Mare et Martin, 2009. Sur les musées anatomiques forains, Christiane Py, Cécile Vidart, «Les musées d'anatomie sur les champs de foire", Actes de la recherche en sciences sociales, vol. 60, nov. 1985, p. 3-10.

11. Sur ces dangers, voir Denis Canguilhem, Le Merveilleux scientifique. Photographie du monde savant en France 1844-1918, Paris, Gallimard, 2004. Sur le spectacle ambigu des corps entre art et médecine : Jean Clair, Jean-Pierre Changeux, L'Âme au corps : arts et sciences, 1793-1993. Cat. exp., Paris, Grand Palais, 19 octobre 1993-24 janvier 1994, Paris, Réunion des Musées nationaux/ Gallimard/Electa, 1993.

12. Sur l'histoire de la céroplastie, voir Michel Lemire, Artistes et mortels, Paris, R. Chabaud, 1990 ; Nathalie Latour, Céroplastie. Corps immortalisés, Paris, Éditions du Murmure, 2020.

13. Sur le répertoire de motifs et la notion d'intericonicité, voir Jean-Jacques Courtine, » Corps, discours, images ", Déchiffrer le corps : penser avec Foucault, Grenoble, Jérôme Millon, 2011.

14. Par exemple, René Micha, "Le musée de l'imaginaire ", dans Georges Achten (dir.), Grand Musée Anatomique, ethnologique du Dr. P. Spitzner, Bruxelles, Snoeck-Ducaju, 1980, p. 16-19 ; Suzanne Lilar et Jeannine Paque, Une Enfance gantoise, Bruxelles, Labor, Espace nord, n¹24, 2001 [1976], p. 160-162 ; Patrick Roegiers, Le Mal du pays: autobiographie de la Belgique, Paris, Seuil, 2003, p. 366-369.

15. Catalogue du grand musée d'anatomie du Dr P. Spitzner, Lille, Dubar et Cie, 1895. Nous remercions très vivement Caroline Ducourau, directrice de la culture historique et du patrimoine historique de l'Université de Montpellier, et Marie-Angeline Pinail, régisseuse des collections, pour leur aide et l'amabilité avec laquelle elles nous ont fourni ces images.

16. Joanna Ebenstein, The Anatomical Venus, Londres, Thames and Hudson, 2016.

17. Sur ces hybridations en général, voir Daniel Raichvarg, op. cit.

18. Catalogue..., op. cit., p. 23-24.

19. Anne Carol, «La nudité au XIX siècle: quelques pistes pour l'histoire des pratiques et des sensibilités », Rives, n³0, 2008, p. 25-37, en ligne : https://journals.openedition.org/rives/2303. Par ultra-nudité, nous entendons une nudité qui dévoile ce qui se cache sous la peau.

20. Sylvain Amic et Michel Hilaire, Alexandre Cabanel (1823-1889) : la tradition du beau, Paris, Somogy éditions d'art, 2010, p. 230.

21. Alain Corbin associe le bord de mer et le spectacle du rivage à une charge sexuelle : Alain Corbin, Le Territoire du vide. L'occident et le désir de rivage, Paris, Flammarion, 2010 [1988], p. 195-196.

22. Elisabeth Stephens, "Venus in the archive. Anatomical Waxworks of the Pregnant Body", Australian Feminist Studies, vol 25, $\mathrm{n}^{\circ}$ 64, juin 2010, p. 133-145.

23. Elle interprète cette occultation sous l'angle des découvertes médicales (ovulation spontanée) qui ont rendu superfétatoire l'orgasme féminin dans la fécondation, et dans le cadre plus général d'un nouveau regard sur la gestation. Il peut s'agir plus prosaïquement d'une stratégie visant à désamorcer les soupçons d'immoralité pesant sur ces exhibitions.

24. Catalogue..., op.cit., p.7. Cette hypothèse soulève toutefois deux objections: d'une part, l'absence de représentation de cadavres à proprement parler dans le musée; d'autre part, le discours sur le progrès porté par les collections. 
25. S'y ajoutait une cire représentant « le prince impérial d'Allemagne attaqué par une maladie de la gorge ». Il s'agissait du petit-fils de la reine Victoria, emporté par le croup ou un cancer à 11 ans en 1878.

26. Catalogue..., op. cit., p. 13.

27. La mise en valeur des progrès médicaux passait aussi par des objets : «La transfusion du sang » intégrait l'appareil mis au point par le docteur Roussel, tandis qu'une vitrine exposait « La couveuse d'enfants ", dont le fonctionnement était longuement décrit dans la notice.

28. Gérard Tilles, Daniel Wallach, Le Musée des moulages de l'hôpital Saint-Louis, Paris, Doin-APHP, 1996.

29. Alain Corbin, «Le péril vénérien au début du siècle : prophylaxie sanitaire et prophylaxie morale », dans Lion Murard, Patrick Zylberman (dir.), L'Haleine des faubourgs. Ville, habitat et santé au XIX ${ }^{e}$ siècle, Recherches, $\mathrm{n}^{\circ} 29$, décembre 1977.

30. Sur les stratégies visuelles de dissuasion, voir Anne Carol, « Les médecins et la stigmatisation du vice solitaire (fin XvIII ${ }^{\mathrm{e}}$-début $\mathrm{XIX}^{\mathrm{e}}$ siècle)», Revue d'Histoire moderne et contemporaine, janvier-mars 2002, n 49-51, p. 156-172.

31. Félicien Rops, La Leçon d'hygiène, 1878-1880, aquarelle, gouache, pastel, 21,5 x 14,5 cm, Paris, coll. privée.

32. Bruno Bertherat, » Le kaléidoscope des émotions. L'exposition publique des cadavres à la morgue à Paris au XIX ${ }^{\mathrm{e}}$ siècle ", dans Anne-Claude Ambroise-Rendu, AnneEmmanuelle Demartini, Hélène Eck et Nicole Edelman dir., Émotions contemporaines, XIX ${ }^{e}$ XXI ${ }^{e}$ siècles, Paris, Armand Colin, coll. Recherches, 2014, p. 139-153 ; Emmanuel Taïeb, La Guillotine au secret. Les exécutions publiques en France 1870-1914, Paris, Belin, 2011.

33. Par exemple Sylvie Aubenas, Philippe Comar, Obscénités. Photographies interdites d'Auguste Belloc, Paris, Albin Michel, 2001.

34. Anne Carol, «L'examen gynécologique XVIII ${ }^{\mathrm{e}}-\mathrm{XIX}^{\mathrm{e}}$ siècles : techniques et usages », dans Patrice Bourdelais, Olivier Faure (dir.), Les Nouvelles Pratiques de santé XVIII -XIX ${ }^{e}$ siècles, Paris, Belin, 2005, p. 51-66.

35. Pauline Mortas, Une Rose épineuse. La défloration en France au XIX siècle, Rennes, PUR, 2017. Voir aussi Aïcha Salmon, La Nuit de noces. Une histoire sociale et culturelle de l'intimité conjugale (France, années 1800-1920), thèse de doctorat d'histoire de l'Université de Paris 1 Panthéon-Sorbonne, 2021.

36. Champfleury, «L'homme aux figures de cire ", Les excentriques, Paris, Michel Lévy frères, 1852 [1849], p. 349-373

37. Cité dans Laurence Des Cars (dir.), Gustave Courbet, Paris, Édition de la Réunion des Musées Nationaux, 2007, p. 380.

38. Pascal Blanchard et al. (dir.), Zoos humains et exhibitions coloniales, Paris, La Découverte, 2011.

39. Delphine Peiretti-Courtis, Corps noirs et médecins blancs. La fabrique du préjugé racial, XIXe-XXe siècle, Paris, La Découverte, 2021.

40. La stéatopygie et la macronymphie, sont respectivement la proéminence très accusée des fesses par une masse graisseuse et l'allongement prononcé des petites lèvres.

41. Catalogue..., op. cit., p. 21.

42. François-Xavier Fauvelle, «Les Khoisan dans la littérature anthropologique du $\mathrm{XIX}^{\mathrm{e}}$ siècle. Réseaux scientifiques et construction des savoirs au siècle de Darwin et de Barnum ", Bulletins et Mémoires de la Société d'Anthropologie de Paris, t. XI, 1999, p. 425-471.

43. Robert Bogdan, La Fabrique des Monstres. Les États-Unis et le Freak Show (1840-1940), Paris, Alma Éditeur, 2013 [1988].

44. Jean-Jacques Courtine, «Le corps anormal. Histoire et anthropologie culturelle de la difformité ", dans Jean-Jacques Courtine (dir.), Histoire du corps, t. III : Les mutations du regard : le XX ${ }^{e}$ siècle, Paris, Seuil, 2006, p. 201-262. 
45. Ce n'est qu'au $x^{e}$ siècle que se constitue une petite section explicitement vouée aux «monstres » et contenant une demi-douzaine de pièces, dont les frères Tocci constituent le joyau.

46. En 1902, ces deux enfants font l'objet d'une tentative de séparation par le chirurgien Eugène Doyen à Paris le 9 février. Cette séparation, filmée, fit l'objet d'une médiatisation intense, et le docteur Doyen fut accusé (à tort) d'avoir fait projeter son film à la foire du Trône, ce qui illustre la frontière poreuse entre science et divertissement dans le cas des monstres. Sur les monstres doubles et cette polémique en particulier, voir Anne Carol, «Ordre et désordre des monstres doubles dans les théories et les pratiques médicales ", dans Régis Bertrand, Anne Carol dir. Le monstre humain. Imaginaire et société, Aix-en-Provence, PUP, 2005, p. 119-140, en ligne : https:// books.openedition.org/pup/6832.

47. Sur la querelle des monstres doubles, voir Patrick Tort, L'ordre et les monstres. Le débat sur l'origine des déviations anatomiques au XVIII siècle, Paris, Le Sycomore, 1980, $264 \mathrm{p}$.

48. Jean-Louis Fischer, Monstres. Histoire du corps et de ses défauts, Paris, Syros, 1991.

49. Catalogue..., op. cit., p. 30.

50. Marc Renneville, Le Langage des crânes. Une histoire de la phrénologie, Paris, Les empêcheurs de penser en rond, 2000.

51. Anne Carol, Physiologie de la Veuve. Une histoire médicale de la guillotine, Seyssel, Champ Vallon, 2012

52. Dominique Kalifa, L'Encre et le sang. Récits de crime et société à la Belle Époque, Paris, Fayard, 1995 ; Crime et culture au XIX $X^{e}$ siècle, Paris, Perrin, 2005.

53. Vanessa R. Schwarz, Spectacular realities. Early Mass Culture in Fin-de-siècle Paris, Berkeley, Los Angeles, Londres, University of California Press, 1998, notamment chapitre III.

54. Anonyme, «La guillotine à Lille », Le Petit Parisien :journal quotidien du soir, $\mathrm{n}^{\circ}$ 6385, p. 1.

55. Pierre Spitzner, Catalogue du Musée historique, Lille, P. Lagrange, 1899. Le fils de Spitzner, également prénommé Pierre, est né en 1878.

56. Le musée est déjà décrit comme un objet de nostalgie dans les années 1940 ; lorsqu'il réapparaît à l'aube des années 1980 , son étrangeté est devenue si radicale qu'elle inspire un des derniers textes d'Italo Calvino, "Le musée des monstres de cire », dans Collection de sable, paru en 1984 (Milano, Garzanti).

\section{RÉSUMÉS}

Le musée Spitzner, fondé en 1856, est un des plus célèbres musées anatomiques et forains de la seconde moitié du XIX ${ }^{\mathrm{e}}$ siècle en Europe ; ses collections, principalement constituées de cires, sont souvent analysées aujourd'hui par le prisme d'une esthétique de l'étrange. À travers la présentation de 9 pièces représentatives de ses collections, cet article a pour objectif d'en restituer la dimension pédagogique et de tenter d'en recontextualiser la perception par les contemporains.

The Spitzner Museum, founded in 1856, is one of the most famous anatomical and fairground museums of the second half of the 19th century in Europe; its collections, mainly made up of waxes, are often analysed today through the prism of an aesthetics of the "uncanny". Through 
the presentation of 9 representative pieces from its collections, this article aims to restore the educational dimension and to try to recontextualize the perception of its contemporaries.

INDEX

Mots-clés : musée Spitzner, cires anatomiques, musées forains, hygiène sociale

Keywords : Spitzner Museum, anatomical waxes, funfair museums, social hygiene

\section{AUTEURS}

ANNE CAROL

Aix Marseille Univ, CNRS, TELEMME

\section{BÉATRICE HERMITTE}

Aix Marseille Univ, CNRS, TELEMME 